\title{
Does ACL reconstruction have any influence in meniscal repair? Sports return, quality of life and failure rate. 2-12 years follow up.
}

Juan Miguel Rodríguez-Roiz ( $\nabla$ jmleon42@gmail.com )

Hospital Clinic de Barcelona

\section{Sergi Sastre-Solsona}

Hospital Clinic de Barcelona.

Andrés Combalia Aleu

Hospital Clinic de Barcelona

Dragos Popescu

Hospital Clinic de Barcelona.

Jordi Montañana Burillo

Hospital Clinic de Barcelona.

Research article

Keywords: ACL, meniscal repair,meniscal tear, sports return, meniscus

Posted Date: May 29th, 2020

DOl: https://doi.org/10.21203/rs.3.rs-29938/v1

License: (9) This work is licensed under a Creative Commons Attribution 4.0 International License.

Read Full License 


\section{Abstract \\ Objective}

few studies approach with long follow up meniscal repair at amateur level, specially studying variables as quality of life and failure rate. We study medium to long-term clinical results in patients at amateur sports level, that have required meniscal sutures at our centre, with or without $A C L$ reconstruction. We evaluate the objective function of the knee, as well as the patients' return to sports activities, quality of life and the rate of failed repair and study the possible reasons.

\section{Methods}

We design an observational retrospective study, assessed 92 patients that required a meniscal repair in our centre from april 2007 until april 2018, who regularly perform amateur sports activities (Tegner 4 to 7), minimum follow-up period of 2 years, divided into 2 groups: group 1 isolated meniscal suture (43 cases), group 2 associated to ACL reconstruction (49 cases). Every patient have this tests in 2019: Lysholm and Tegner (validated for spanish) before the injury and after the surgical procedure, motivation to return to sports activity (Likert scale with 3 items: low, regular or high), quality of life (through SF-12 test).

\section{Results}

high return to amateur sports rate (92\%), which was even higher in the isolated reconstruction group in comparison to the group with associated $\mathrm{ACL}$, at a minimun follow-up of 2 years. We have not found statistically significant differences between sports return and: age, gender, injured meniscus, chondral injuries, preoperative Tegner score, or motivation. No significant differences in physical or mental health fields between both groups. Meniscal repair failed in 12 patients $(13 \%$,) and they finally required a partial meniscectomy, as complications just one debridement for superficial infection of ACL donor zone. Higher rate of failure in isolated bucket-handle tear injuries $(p<0.0062)$. We have not found a statistically significant association between the other variables studied.

\section{Conclusions}

$92 \%$ of sports return and low meniscal repair failure rate, even lower when is associated to ACL reconstruction.

\section{Background}

The meniscus plays a fundamental role in knee biomechanics, acting not only as a joint shock absorber, but also have propioceptive, lubricating and stability functions. It is well known that after a partial 
meniscectomy, the incidence rate of knee osteoarthritis increases [1]. Considering this, it is understandable that meniscal repair is the gold standard treatment for damaged menisci in young patients, in repairable cases.

Traumatic meniscus injuries are often associated with anterior cruciate ligament tears, chondral lesions, medial collateral ligament injuries, among others, which can worsen the knee's functional prognosis. In these cases, the objective must be recover function and also the patient's quality of life, a factor which is becoming more and more important and which few studies on this topic take into consideration $(1,2)$

$30-60 \%$ of anterior cruciate ligament $(\mathrm{ACL})$ tears are associated with meniscal or chondral injuries at the time of their index reconstruction $[3,4,5]$, specifically the lateral meniscus the most common. The lateral meniscus has different structural and functional properties than the medial meniscus than explains this situation, first a lack of attachments to the popliteal hiatus and collateral ligament and a relative greater mobility, that reduce the risk of ruptures, thats the reason this meniscus is rarely affected in a stable knee (6)

Regarding the role of the menisci as secondary stabilizers within the knee, it is well known that the clinical results of anterior cruciate ligament (ACL) reconstruction are markedly impaired by the presence of concurrent meniscal injury. Anterior drawer was not increased significantly after medial meniscectomy when the further increase in tibial anterior translation of up to $5.8 \mathrm{~mm}$ (7), thus highlighting the importance of the meniscus-meniscal ligament construct as a secondary joint stabilizer in synergy with the ACL.

Similarly, the meniscus act as secondary restraints to tibial internal-external rotation. Wang and Walker (8) found that meniscectomy increased the range of rotation $5^{\circ}$ from a mean of $18-23^{\circ}$, at $0.5 \mathrm{~N} \mathrm{~m}$ torque. The laxity was further increased if the ligaments were excised: removal of ACL and PCL (posterior cruciate ligament) increased laxity $23^{\circ}$, while removal of both role of this ligament is unclear and its influence on the overall meniscal biomechanics remains elusive. It has been reported to serve in some specimens as the primary attachment of the anterior horn of the medial meniscus and proposed to act as a restraint to anterior subluxation and excessive posterior translation when the menisci are under load (9). Finally, it is also believed that it acts as a tie between the menisci that controls their relative positioning on the tibial plateau when the tibia rotates.

Failure rates of meniscal repair is "low" ( $<15 \%)$ and is lower when is associated to ACL reconstruction [6]. It has been reported that $A C L$ reconstruction provides a positive biomechanical environment for the meniscus to heal (6). The clinical picture of this situation is controversial.10 Several studies have reported both higher and similar healing rates with concomitant ACL reconstruction (11-13)

The most of literature about meniscal repair and ACL reconstruction is focused in the professional athlete, that is not the "day by day" patient in clinics worldwide, so maybe the results that we found in literature couldnt be extrapoled to the most of the cases, that situation can raise high expectations fot both surgeon and patient. 
The medium follow-up of most of studies about this topic is around 2-5 years, so the long term results, specially in amateur level, is not studied enough and these time periods may represent a range in which also simple partial meniscectomy usually provides acceptable results

Today,variables as quality of life and patients satisfaction, are studied much more than 10 years ago. But about meniscal repair/ACL reconstruction there is just a few studies that review this important outcome.

The purpose of the present study was investigate the medium to long-term clinical (2-12 years) results of patients at amateur sports level, who underwent isolated meniscal repair or combined with ACL reconstruction, specifically:

- Determine rate of amateur sports return. We use Tegner scale for determine the sports than patient practice before injury, and the actual sport, and determine not only is the patient returns, also if returns to the same tegner sports level. We also study if there is a difference in sports return between patients than required an isolated meniscal repair, and the patients that also required an ACL reconstruction. Patients motivation for sports return is also measured.

- Functional results measured by Lysholm score

- Quality of life and satisfaction with the treatment performed, using SF-12 test, evaluating mental and physical scale.

- Failure rate of meniscal repair and possible reasons to explain that cases of failure, we diagnosed meniscal repair failure combining clinical and MRI results. We take that patients with a failed meniscal repair and study the outcomes after the revision surgery, if needed.

Our hipothesis is that patients in both groups (isolated meniscal repair, or with an ACL reconstruction) will have high sports return values, with higher functional and quality of life outcomes in the patients with isolated meniscal repair than the ACL reconstruction group, but this group will have less meniscal repair failure. Also we think that higher motivated patients will have higher return to sports rates and in less time.

\section{Methods}

A consecutive series of traumatic meniscal tear cases operated at our centre with arthroscopic meniscal suture with or without ACL reconstruction were assesed retrospectively, between April 2007 and April 2018 , a total of 380 patients

\section{Inclusion criteria}

1) Patients between the ages of 13 and 55 who regularly perform recreational sports activities (Tegner 4 to 7 , minimun 3 times/week), with a minimum follow-up period of 2 years 
2) Patients were assessed through online/in-person questionnaires, filled in between April and November 2019, including the following tests: Lysholm and Tegner (validated for spanish) before the injury and after the surgical procedure, motivation to return to sports activity (Likert scale with 3 items: low, regular or high), quality of life (through SF-12 test), and meniscal repair failure (all cases diagnosed by a MRI with compatible symptoms, and a second surgery was required). Data about type of meniscal injury and type of repair, was obtained from surgical report.

3) An observational retrospective study was conducted, according to the WMA Declaration of Helsinki, and was reviewed and approved by our institutional review board (document attached).

\section{Exclusion criteria}

1) Patients with less than 13 years, or more than 55 years at the time of surgical intervention

2) ACL reconstructions alone or with a meniscectomy (no repair)

3) Sedentary patient or professional athlete

4) Prior surgeries on the injured knee

5) Degenerative chondral injuries (Outterbridge III or more), we just include maximum Outterbridge type II chondral injuries.

6) Discoid meniscus or multiligamentous injuries

7) Postoperative follow-up of less than one year, or patients impossible to locate or that doesnt want to participate in the study.

8) Patients that need meniscal root reinsertion, or meniscal ramp reconstruction.

Finally, 92 patients were included in the study, divided into 2 groups: group 1 corresponded to the patients that required isolated meniscal suture (43 cases), group 2 included the cases that required meniscal suture associated to ACL reconstruction (49 cases).

Group 1 (isolated meniscal suture) included 43 patients, 28 men and 15 women, with an average age of 32.18 years (13-47 years), with an approximate follow-up period of 7 years (2-12.5 years). The interval between injury and surgical intervention was an average of 11.74 months (1-50 months). An average of 2 meniscal sutures (1.93) was used in each case (1 to 3 ) (Table 1 ).

Group 2 (ACL reconstruction plus meniscal suture) included 49 patients, 35 men and 14 women, with an average age of 29.71 years (16-54 years), with an approximate follow-up period of 6 years (212.5 years). The interval between injury and surgical intervention was an average of 16.48 months (280 months). An average of 2 meniscal sutures (1.73) was used in each case (1 to 3 ) (Table 1 ). 


\subsection{SURGICAL TECHNIQUE}

All patients were evaluated, as usual, by an internal medicine or anesthesiologist before the surgical procedure. Surgical procedure was performed in supine position, under spinal anesthesia plus endovenous sedation, with the affected knee flexed and with a pneumatic tourniquet cuff on the ipsilateral thigh.

In all cases, arthroscopic meniscal suture was performed through 2-3 conventional arthroscopic portals (anterolateral, anteromedial and accessory anteromedial portals), and the suture technique of choice was the Fast-Fix type all-inside suture (Smith-Nephew) and outside-in suture specifically for body and anterior horn injuries (less usual). Lenght of the suture was usually $16 \mathrm{~mm}$ for internal meniscus and $18 \mathrm{~mm}$ for lateral meniscus. In all the cases with internal meniscus injury we perform a "pie-crust" of the superficial internal collateral ligament to have more space and avoid iatrogenical chondral injuries. We usually made a concious debridement of meniscal borders before the repair, and also microfractures in the medial femoral condyle to favor the meniscal healing process.

In the cases that required $A C L$ reconstruction, the hamstring autograft was the graft of choice in our study. In all cases, we initially obtained the autograft following the traditional technique with a surgical incision near the anterior tibial tuberosity in proximal-medial tibia. Graft was measured and bony tunnels were drilled based in that size. An anatomical reconstruction technique was used in all cases.

Femoral tunnel is always drilled in knee hiperflexion $\left(>110^{\circ}\right)$ using anteromedial portal and using landmarks to select the anatomical position. In some cases we use a lateral knee X-ray intraoperatively to confirm the position. Endobutton system (Smith-Nephew) was used in all cases for femoral fixation, and for tibial fixation we use bioreabsorbable screws always tightened in knee semi-flexion $\left(15^{\circ}\right)$ and performing a posterior draw force. Surgical closure with stitches as usual, and we usually use 2 drainages for 24 hours, one for graft donor zone and another intra-articular.

\subsection{Rehabilitation and postoperative protocol}

Patients who underwent isolated meniscal repair and those who underwent meniscal repair with ACL reconstruction completed the same postoperative rehabilitation programme.

The patients were discharged home 24 hours after surgery. In all cases, a Don-Joy knee brace was placed maxium at $60^{\circ}$ of flexion. For the first 2 weeks, patients make non weight-bearing with help of 2 crutches and begin immediate rehabilitation treatment: local anti-inflammatory treatment + progressive passive knee kinesiotherapy + isometric quadriceps exercises. After 2 weeks, they were authorised to begin walking with progressive partial weight-bearing. One month after surgery, they were authorised to flex to $90^{\circ}$ for 1-2 weeks, as well as full weight-bearing. On week 6 after surgery, the brace was maintained without flexion limitation and they began walking independently without crutches.

\subsection{OUTCOME MEASURES}




\section{LYSHOLM SCORE}

The Lysholm Knee Scoring Scale has an extended use beyond evaluating outcomes of knee ligament surgery. It can also be used for meniscal tears, knee cartilage lesions, osteochondritis dissecans, traumatic knee dislocation, patellar instability, patellofemoral pain, and knee osteoarthritis.

Consist of eight items that measure: pain (25 points), instability (25 points), locking (15 points), swelling (10 points), limp (5 points), stair climbing (10 points), squatting (5 points), and need for support (5 points). Every question response has been assigned an arbitrary score on an increasing scale. The total score is the sum of each response to the eight questions, and may range from 0-100. Higher scores indicate a better outcome with fewer symptoms or disability.

A study carried out by Briggs et al. showed the Lysholm questionnaire to have acceptable test-retest reliability, floor and ceiling effects, criterion validity, construct validity, and responsiveness to change [143]. Finally, the questionnaire itself is relatively easy for patients to complete and does not have a complicated scoring methodology.

\section{TEGNER ACTIVITY SCORE}

The Tegner activity scale was first described in 1985 and initially designed for physician administration after ACL and meniscal injuries. To date, the Tegner activity score has been a frequently used patientadministered activity rating system for patients with various knee disorders

The Tegner Activity Scale classifies both sports and work activities into one questionnaire using an 11level gradient. Competitive sports make up the top three levels (levels $10-8$ ), competitive and recreational sports categories both appear in level 7, and "other recreational sports" make up level 6. Levels 5 through 1 combine work and sports together, and level 0 indicates sick leave or disability because of the knee condition.

\section{MOTIVATION TO SPORTS RETURN}

Motivation involves the internal processes that give behaviour its energy and direction. Motivation originates from a variety of sources (needs, cognitions and emotions) and these internal processes energize behaviour in multiple ways such as starting, sustaining, intensifying, focusing, and stopping it.

We use a unipolar Likert scale question type for measure this value. For the question: ¿How much motivated you are for return to your sports activities?. The three options were: 1) Low motivated, 2) More or less motivated, 3) Highly motivated.

\section{SF-12 HEALTH SURVEY TEST}

The 12-item Health Survey (SF-12) was developed as a shorter alternative to the SF-36 for use in largescale studies, and its reliability and validity have been documented [16]. All 12 items are used to calculate the physical and mental component summary scores (PCS-12and MCS-12) by applying a scoring 
algorithm empirically derived from the data of a US general population survey. The SF-12 test ranges from 0 to 100, where a higher score implies a better quality of life with regard to health $[17,18]$.

\subsection{STATISTICAL ANALYSIS}

Statistical analysis were performed using SPSS version 23.0 (IBM. New York, USA). The following descriptive variables were calculated: mean, standard deviation, median, range, frequency and percentage. The normality of the distribution for measured outcome variables was evaluated using the Kolmogorov-Smirnov test. An independent sample t-test was used to detect differences between preoperative and postoperative outcome scores for all outcomes measured. The alpha level of significance was set at $p<0.05$.

\section{Results}

In 92 cases, we have 7 meniscal repair failures (16.27\%) in group 1 (isolated meniscal repair) and 4 failures $(8.16 \%)$ in group 2 (ACL reconstruction plus meniscal repair); the mean time until a tear reoccurred was 2.7 years (range, 1.3-4.4 years) and 5.0 years (range, $0.8-7.5$ years), respectively. When analysing subgroups of patients with recurring tears compared to the group of patients that did not require another surgery, we observed that those with a higher frequency of bucket-handle injuries were the ones that failed. No differences have been found with regard to the presence of an ACL injury or the number of sutures used. (Table 2).

\subsection{Lysholm score}

Group 1 (isolated meniscal suture) included 43 patients, 28 men and 15 women, with an average age of 32.18 years (13-47 years), with an approximate follow-up period of 7 years (2-12.5 years). The interval between injury and surgical intervention was an average of 11.74 months (1-50 months) (Table 1). The average Lysholm test was 89.34 (58-100), indicating excellent results (95-100) in 15 patients (35\%), good results (84-94) in 20 cases (47\%), fair results (65-83) in 6 cases (14\%), and poor results $(<64)$ in only 2 cases $(4.65 \%)$ (Table 1$)$.

Group 2 (ACL reconstruction meniscal suture) included 49 patients, 35 men and 14 women, with an average age of 29.71 years (16-54 years), with an approximate follow-up period of 6 years(1-

12.5 years). The interval between injury and surgical intervention was an average of 16.48 months (280 months). The average Lysholm test was 84.69 (15-100), indicating excellent results (95-100) in 10 patients (20.40\%), good results (84-94) in 27 cases (55.1\%), fair results (65-83) in 8 cases $(16.32 \%)$, and poor results $(<64)$ in only 4 cases $(8.16 \%)$ (Table 3$)$.

We did not find statistically significant differences between both groups $(\mathrm{p} 0.10)$.

\subsection{Tegner activity score}


In group 1, with an average follow-up period of 7 years (2-12.5 years), patients presented a discrete increase in their baseline Tegner score from $5.11(+/-1.37)$ to $5.46(+/-1.44)$. Out of 43 patients, only 2 did not return to exercise.

In group 2, with an average follow-up period of 6 years (1-12.5 years), patients presented a discrete decrease in their baseline Tegner score from $6(+/-0.98)$ to $5.31(+/-1.43)$. Out of 49 patients, 5 did not return to exercise.

We were not able to find statistically significant differences with regard to the pre-and postoperative Tegner score in either group ( $\mathrm{p}$ 0.59), or regarding giving up amateur sports activities (Table 3 ).

\subsection{SF-12 Health Survey Test}

In group 1, all patients except three declared they were very satisfied with the postoperative outcome in both physical and mental health fields (ranging from 30.8 to 63.5 and 32 to 68 , respectively), and further indicated that if it were required again, they would undergo the same procedure under the same circumstances (Table 3).

In group 2, all patients except six were very satisfied with the postoperative outcome in both physical and mental health fields (ranging from 26.93 to 63 and 26 to 68,respectively) (Table 3).

No significant differences were found between both groups with regard to physical or mental health fields (Table 3).

\subsection{COMPLICATIONS AND RE-INTERVENTIONS}

The principal cause for re-intervention in our series was due to meniscal suture failure, and one case of local debridement (donor zone) with no other note worthy complications requiring further surgery (septic arthritis, ACL re-rupture, etc.). We considered meniscal suture failure in patients with persistent or recurrent symptoms attributable to meniscal injury worthy of another surgical intervention. In all cases, the clinical diagnosis was confirmed with an MRI and a partial arthroscopic meniscectomy was subsequently performed on the failed repair area. In group 1, out of 43 patients, the meniscal suture failed in 7 cases (16.28\%); 6 cases of medial meniscus repair ( 2 bucket-handle tears), 1 case of lateral meniscus repair. There were 4 cases of all-inside suture, and 3 cases of hybrid technique (all-inside and inside-out). In 4 cases $2-3$ sutures had been used, in 2 cases, 4 sutures or more, and in 1 case, one suture. The average time between both surgeries was 3.17 years ( 8 months- 11 years), with an average age group of 33 years (17-47 years).

In group 2, out of 49 cases, the meniscal suture failed in 5 cases $(10.20 \%) ; 2$ cases of lateral meniscus repair, 3 cases of medial meniscus repair ( 1 bucket-handle tear). In all cases an all-inside suture had been performed: in 1 case with 1 suture, and in 4 cases with 2 to 3 sutures. The average time between both surgeries was 3.6 years ( $1-7$ years), with an average age group of 26 years (18-32 years). 
All cases of meniscal suture failure required a partial meniscectomy, and were reevaluated at 2 years postop with the same initial tests.

Cumulative incidence values for failure were determined along with their $95 \%$ Cls for the various demographic predictors analysed during Cox proportional hazards regression (Table 2). No single variable was found to predict increased failure incidence over time.

\section{Discussion}

\subsection{MENISCAL REPAIR OUTCOMES}

Good clinical results after meniscal repair with or without ACL reconstruction were obtained, with a low suture failure rate and few complications. We found a statistically significant difference $(p<0,001)$ in meniscal repair failure between groups. In group 1 (isolated meniscal repair) 7 of 43 repairs fail and required a partial meniscetomy, in group 2 (ACL reconstruction associated) just 4 of 49 , so ACL reconstruction must have a "protective influence" on meniscal repair. A systematic review by Paxton et al (19) showed an overall reoperation rate of $24 \%$ after meniscal repair compared with $14 \%$ when performed in conjunction with ACL reconstruction, and this relation was maintained even when analyzed by specific repair methods and devices.

In a study of reoperations after meniscal repair, withand without concomitant ACL reconstruction, Wasserstein et al (20) concluded that meniscal repair performed in conjunction with ACL reconstruction carries a $7 \%$ absolute and $42 \%$ relative risk reduction of reoperation after 2 years compared with isolated meniscal repair.

Potential explanation for better results when meniscal repair is associated to ACL reconstruction, both in literature and in our study, could be the blood and bone marrow cells that are liberated after drilling bony tunnels, the biomechanical stability that $\mathrm{ACL}$ reconstruction gives to the knee explains that results, the relatively limited patient activity, and maybe the less agresive rehabilitation after combined procedures. Also we found a higher failure for internal meniscus repair (9 failures in 66 repairs) than external ( 3 fails of 24 repairs), not significant difference, but can be explain by the stability of meniscus, external meniscus have higher mobility upon the tibial plateau so have more resistance to shear forces $(21,22)$.

The outside-in suture has traditionally been considered the gold standard of meniscal repair, although recent studies have found similar results with all-inside suture techniques, and, specifically in buckethandle tears, no clinically significant differences have been found in the medium term between all-inside or inside-out meniscal sutures $[10,11]$. Biomechanical studies show the same resistance to load-bearing in both repair techniques $[22,23]$.

The literature reports an incidence of meniscal retears ranging from $20 \%$ to almost $40 \%$. The risk factors for meniscal retears were the size (length of the tear), the presence of a complete tear, and a positive pivot-shift test finding (residual instability). (24) 
In this study the meniscal repair failed in 12 patients (13\%). We observed a higher rate of suture failure in isolated bucket-handle tear injuries in comparison to other types of meniscal injuries, which is a statistically significant difference $(p<0.0062)$. We didnt found clinically significant differences with regard to recurring tears when comparing all-inside technique with cases using the hybrid technique (outside-in + all-inside). Statistically significant association between the other variables studied and meniscal repair failure were not found: age, gender, knee laterality, injured meniscus, number of sutures used, preoperative Tegner score, time between injury and surgical intervention or chondral injuries (Table 2).

Traditionally, many experts consider age to be a key factor, ruling out the possibility of meniscal repair in patients over 50 years of age. A recent meta-analysis did not find differences between repair and recurring meniscal tear rates in patients over 40 years of age in comparison to younger patients [25]. We agree with recent literature; we have not observed statistically significant differences between the patient's age and the failure rate of meniscal repair (Table 2).

The type of repair is also important, with several studies proving higher resistance and lower recurring tear rates in vertical sutures compared to horizontal sutures $[2,8,14]$. Indeed, perhaps this is one of the reasons behind the low recurring tear rate in our series, since in almost all cases we tended to use vertical sutures.

In all the patients who presented a meniscal suture failure that required partial meniscectomy, we observed a statistically significant $(p<0.001)$ logical decrease in the Tegner scale (with regard to the initial preoperative score), and an equally lower return rate to sports activities. Nevertheless, when assessing medium to long term, we observed in our series that from functional (Lysholm) and quality-oflife (SF-12) point of view, patients in this group ended up with very similar results to those with a successful meniscal suture. For this reason, we consider selective partial meniscectomy to be a valid option to be considered in cases of failed suture.

\subsection{SPORTS RETURN}

Returning to sports activities is one of the fundamental objectives of meniscal reconstructions, with or without ACL reconstruction. The literature describes a very high percentage of patients that return to sports activities satisfactorily $[26,27]$. Our series also revealed a high return rate $(92 \%)$, which was even higher in the group 1 (isolated meniscal repair) in comparison to group 2(ACL associated) (Table 4), which can be explained by the further complexity of ACL injuries, but no statiscally diference for this result $(p 0,38)$.

Known factors affecting sports return include graft type, patient age, baseline activity level, sport type, and athletic experience within the sport.

We have not found statistically significant differences between returning to sports activities and: age, gender, injured meniscus, lysholm, chondral injuries, preoperative Tegner score, or motivation to return to sports activities (Table 4). 
As a curiosity, we find higher Lysholm medium score in patients than abandonded sports $(91,5)$ than who returned (85), difference no statiscally significant (Table 4, p 0,51). This shows that sports return is somewhat complex, and need more than just a good functional knee, motivation and another psychosocial factors made an important influence. (28)

Preop and postop Tegner score with small diference in both groups and no statiscally significant difference (p 0,59) (Table 3), Tegner activity scores decrease specially in group 2 (from $6+/-0,98$ to 5,31 $+/-1,43$ ) for reasons unrelated to the potential function of the knee (we obtained high lysholm scores postop), that is, by factors unrelated to knee function such as a different social setting in midlife with less time for amateur sports and more focus on family and career.

\subsection{FUNCTIONAL KNEE RESULTS}

We use Lysholm test for measure objectively the function of the knee. Group 1 (isolated meniscal repair) have higher medium Lysholm $(89,34)$ than group 2 (meniscal repair with ACL reconstruction) with 84.69 (Table 3), but the small diference was not statiscally significant ( $p 0,10)$. Difference can be easilly answered because the association between ACL tear and meniscus usually means a higher joint damage and a more complex surgery and rehabilitation process, than an isolated meniscal tear.

We obtain similar Lysholm scores than literature. Zheng X et al (29) reported Lysholm values of $87.7+8.5$ with a medium follow-up of 2 years in ACL reconstruction with autograft. Shirish et al (30) found a medium lysholm score of. 91,4 at 2 years follow up of meniscal repair and ACL reconstruction.

\subsection{QUALITY OF LIFE}

Our study revealed no statistically significant difference both physical and mental areas from SF-12 ( $p$ 0,97 and $p 0,30$ ) between the group of patients with isolated meniscal suture or ACL reconstruction when it comes to quality of life (SF-12) with similar results, with little influence from chondral injuries also, although it must be noted that our study only included Outterbridge stage 2 chondral injuries at the most (Table 2). Chondral injuries at the time of ACL reconstruction have more medium-term impact on quality of life (quantified by SF-12) and functional recovery (WOMAC) than meniscal injuries; subsequently, patients with chondral injuries also have worse results for these variables $[6,18,19,31]$.

Fuch et al found high outcomes in quality of life in isolated meniscal repair, with a follow up of 3 years, measured by KOOS QOL, with a medium score of $81.8 \pm 12.1$. (32)

In a recent retrospective study by Cinque et al (33), in 85 patients with ACL reconstruction with or without meniscal repair, they found similar outcomes in quality of life than our study, using SF-12 they found in physical components medium value of 52,8 , and in mentall components they found 53,2 . And also they didn't found a statiscally significant difference between ages and quality of life outcomes.

\subsection{MOTIVATION FOR SPORTS RETURN}


Motivation for sports return is fundamental in the rehabilitation of this knee injuries, because is a hard process,both physically and mentally, so if the patient is "goal oriented" to come back to sport, is pretty sure this gonna boost the process. The problem is thay maybe highly motivated athletes can push too further the process and maybe early overload the knee and potentially damage the meniscal repair. We didnt find statiscally significant difference between motivation and meniscal succesfull repair or failure (p 0,34) (Table 2).

51 of the 53 highly motivated patients return to sports, but 7 of 8 low motivated also return. We didnt found either a statiscally significant difference $(p 0,18)$

Brewer et al (34) prospectively examined the relationship between psychological factors and rehabilitation outcomes after ACL reconstruction in 95 patients. Self-motivation, athletic identity, and psychological distress were significant preoperative predictors of objective outcomes such as knee laxity. However, postoperative rehabilitation adherence did not affect rehabilitation outcomes.

Nwachukwu et al (35) in a recent systematic review find that the ideal psychological measure of sports return should consider factors including willingness, motivation, and fear of returning both preoperatively and postoperatively.

More studies are necessary to establish the validity and general usability of such questionnaires in patient populations of different sporting activity levels

\subsection{LIMITATIONS}

The limitations of our study need to be acknowledged. First, the strict inclusion criteria meant that the cohort sizes were fairly small. Larger sample sizes would be required to match patients based on additional criteria, such as number of sutures.

The study was retrospective in nature, and thus, analysis was limited to the data available in the medical record database, and with potential for selection bias. Second we define a meniscal succesfull repair as the abscence of meniscal symptoms or a normal MRI, but we dont have second-look arthroscopy confirmation, this was only performed in the cases than repair failed.

Third we dont use objective probes (as KT-1000, etc) to test the knee stability, specially after ACL reconstruction, we just use Lysholm as the indicator for instability. We asume that sports return and ausence of symptoms is good enough, but studies with larger sample of amateur sports players, and more objective test will be needed to confirm our results. Another weakness of the study is the lack of radiographic evaluations that might have correlated with the patient reported outcome scores.

Despite these limitations, our study focused specifically on the isolated repair of meniscal tears in ACLintact knees versus repair with concomitant ACL reconstruction at amateur level.

\section{Conclusions}


In our study, patients that required meniscal suture presented good clinical progress, both from the point of view of returning to sports activities, quality of life (measured by SF-12), and the functional condition of the knee in the medium and long term. The failure rate of meniscal suture is relatively low $(12 \%$ in our study), and is even lower when associated to ACL reconstruction, so this must have a protective function from a mechanic and also biological way, we found higher meniscal repair failure in isolated internal meniscus repair than external meniscus, and this specially in bucket-handle injuries. Age and time from injury until surgery doesnt seems to influence in meniscal repair failure.

\section{Abbreviations}

ACL

ANTERIOR CRUCIATE LIGAMENT

\section{Declarations}

- Availability of data and materials: not applicable

- Acknowledgements: not applicable

-Authors contributions:

JMRR determines the idea and design of work, also acquisition of data, data analysis, work with SPSS, and intensive literature review

DP obtained the cases, made intensive literature review

JMB helps with the design of work, with the statistics analysis and interpretation of data, and used software SPSS.

SS obtained the cases, helps with the idea and design of work

AC helps with the idea and design of the study, also provides the university resources for literature review.

Each author read, revised and approved the manuscript.

- Ethics approval and consent to participate informed consent for participation in the study from patients under 16 years old, was obtained from their parent or guardian.

This study was performed in accordance with the Declaration of Helsinki and have been approved by the hospital ethics committee with data january 2019 (document attached).

- Competing interest: no author have any kind of competing interest.

- Funding: no funding available for this study 
- Consent for publication: not applicable.

\section{References}

1. Barber FA. Herbert MA, Richards DP. Load to failure testing of new meniscal repair devices. Arthroscopy 2004;20(1):45-50.

2. Barber FA. Herbert MA, Schroeder FA. Aziz-Jacobo J, Sutker MJ. Biomechanical testing of new meniscal repair techniques containing ultra high-molecular weight polyethylene suture. Arthroscopy 2009; 25(9):959-967.

3. Boenisch UW, Faber KJ, Ciarelli M, Steadman JR, Arnoczky SP. Pull-out strength and stiffness of meniscal repair using absorbable arrows or ti-cron vertical and horizontal loop sutures. Am J Sports Med 1999;27:626-31.

4. Cinque ME, Chahla J, Mitchell JJ, et al. Influence of Meniscal and Chondral Lesions on PatientReported Outcomes After Primary Anterior Cruciate Ligament Reconstruction at 2-Year Follow-up. Orthop J Sports Med. 2018;6(2)..

5. Everhart JS. Higgins JD, Poland SG. Abouljoud MM, Flanigan DC. Meniscal repair in patients age 40 years and older: A systematic review of 11 studies and 148 patients. Knee. 2018.;25(6):1142-1150..

6. Haklar U, Donmez F, Basaran SH, Canbora MK. Results of arthroscopic repair of partial- or fullthickness longitudinal medial meniscal tears by single or double vertical sutures using the inside-out technique. Am J Sports Med 2013;41(3):596-602.

7. Allen CR. Wong EK, Livesay GA. Sakane M, Fu FH, Woo SL.Importance of the medial meniscus in the anterior cruciate ligament-deficient knee. J Orthop Res. 2000 Jan;18(1):109 - 15.

8. Wang CJ, Walker PS. Rotatory laxity of the human knee joint. J Bone Joint Surg Am. 1974; 56: $161-$ 170.

9. Nelson EW, LaPrade RF. The anterior intermeniscal ligament of the knee: an anatomic study. Am J Sports Med 2000; 28:74-76..

10. Epple JJ, Dunn WR, Wright RW. Meniscal repair outcomes at greater than five years: a systematic literature review and meta-analysis.J Bone Joint Surg Am 2012;94(24):2222-2227.

11. Uzun E. Misir A, Kizkapan TB. Ozcamdalli M, Akkurt S. Guney A. Factors affecting the outcomes of arthroscopically repaired traumatic vertical longitudinal medial meniscal tears. Orthop J Sports Med.2017;5(6)..

12. Vaquero-Picado A, Rodríguez-Merchán EC. Arthroscopic repair of the meniscus: Surgical management and clinical outcomes. EFORT Open Rev.2018; 8;3(11):584-594..

13. Wasserstein D, Dwyer T, Gandhi R, Austin PC, Mahomed N, Ogilvie-Harris D. A matched-cohort population study of reoperation after meniscal repair with and without concomitant anterior cruciate ligament reconstruction. Am J Sports Med. 2013;41: 349-355..

14. Reliability, Validity, and Responsiveness of the Lysholm Knee Score and Tegner Activity Scale for Patients with Meniscal Injury of the Knee. Karen K. Briggs, Mininder Kocher S. William G. Rodkey, J. 
Richard Steadman, J Bone Joint Surg Am 2006, 88 (4) 698-705..

15. Ross M. Relation of implicit theories to the construction of personal histories. Psychological Review $1989,96,341-357$.

16. Ware JE. Kosinski M, Keller SD A 12-Item Short-Form Health Survey: construction of scales and preliminary tests of reliability and validity. Med Care 1996; 34:220-233.

17. Hagino T. Ochiai S, Senga S. Yamashita T, Wako M. Ando T, Haro H.Meniscal tears associated with anterior cruciate ligament injury. Arch Orthop Trauma Surg 2015; 135(12):1701-1706. .

18. Han SB, Shetty GM, Lee DH, Chae DJ, Seo SS, Wang KH, Yoo SH, Nha KW. Unfavorable results of partial meniscectomy for complete posterior medial meniscal root tear with early osteoarthritis: a 5to 8-year follow-up study. Arthroscopy 2010. (26):p. 1326-32..

19. Paxton ES. Stock MV, Brophy RH. Meniscal repair versus partial meniscectomy: A systematic review comparing reoperation rates and clinical outcomes. Arthroscopy 2011;27:1275-1288.

20. Wasserstein D, Dwyer T, Gandhi R, Austin PC,Mahomed N, Ogilvie-Harris D. A matched-cohort population study of reoperation after meniscal repair with and without concomitant anterior cruciate ligament reconstruction. Am J Sports Med 2013;41:349-55.

21. Popescu D. Sastre S, Garcia Al. Tomas X, Caballero M. MR-arthrography assessment after repair of chronic meniscal tears. Knee Surg Sports Traumatol Arthrosc 2015;23(1):171-7..

22. Rimmer MG. Nawana NS, Keene GC. Pearcy MJ. Failure strengths of different meniscal suturing techniques. Arthroscopy 1995; 11:146-50.

23. Miao Y. Yu JK, Ao YF. Zheng ZZ, Gong X. Leung KK. Diagnostic values of 3 methods for evaluating meniscal healing status after meniscal repair: comparison among second-look arthroscopy, clinical assessment, and magnetic resonance imaging. Am J Sports Med 2011;39:735-742.

24. Matsushita T. Nagai K, Araki D, et al. Factors associated with the status of meniscal tears following meniscal repair concomitant with anterior cruciate ligament reconstruction. Connect Tissue Res 2017; 58:386-392..

25. Nepple JJ. Dunn WR, Wright RW. Meniscal repair outcomes at greater than five years: a systematic literature review and meta-analysis. J Bone Joint Surg Am 2012;94:2222-7..

26. Rodríguez-Roiz JM. Caballero M, Ares O. Sastre S, Lozano L. Popescu D. Return to recreational sports activity after anterior cruciate ligament reconstruction: a one- to six-year follow-up study.Arch Orthop Trauma Surg 2015.;135(8):1117-22..

27. Samuelsen BT, Johnson NR, Hevesi M, Levy BA, Dahm DL, Stuart MJ, Krych AJ. Comparative Outcomes of All-Inside Versus Inside-Out Repair of Bucket-Handle Meniscal Tears: A PropensityMatched Analysis Orthop J Sports Med 2018. 15;6(6)..

28. Lentz TA, Zeppieri G Jr, George SZ, et al. Comparison of physical impairment, functional, and psychosocial measures based on fear of reinjury/lack of confidence and return-to-sport status after ACL reconstruction. Am J Sports Med 2015;43:345-53. 
29. Zheng X, Hu Y, Xie P, Li T, Feng YE, Gu J, Gao S. Clinical outcomes and second-look arthroscopic findings of anterior cruciate ligament reconstruction with autograft, hybrid graft, and allograft. $J$ Orthop Surg Res. 2019 Nov 21;14(1):380.

30. Pathak S. Abhinav Bharadwaj MS, Prateek Patil MS, Sudarshan Raut MS, M.S., Srikanth RV. Functional Outcomes of Arthroscopic Combined Anterior Cruciate Ligament Reconstruction and Meniscal Repair: A Retrospective Analysis. Arthroscopy, Sports Medicine, and Rehabilitation 2020; 2(2): e71-e76..

31. Michalitsis S, Vlychou M, Malizos KN, Thriskos $P$, Hantes ME. Meniscal and articular cartilage lesions in the anterior cruciate ligament-deficient knee: correlation between time from injury and knee scores. Knee Surg Sports TraumatolArthrosc 2015;23(1):232-9.

32. Fuchs A, Ferdinand Kloos, Gerrit Bode, Kaywan Izadpanah, Norbert P. Südkamp, Matthias J. Feuch. Isolated revision meniscal repair - failure rates, clinical outcome, and patient satisfaction. BMC Musculoskeletal Disorders (2018) 19:446.

33. Cinque ME. Chahla J, Moatshe G. DePhillipo NN, Kennedy NI. Godin JA, Robert F.LaPrade. Outcomes and Complication Rates After Primary Anterior Cruciate Ligament Reconstruction Are Similar in Younger and Older Patients. Orthop J Sports Med. 2017 Oct 2;5(10).

34. Brewer BW. Cornelius AE, Van Raalte JL, Tennen H. Armeli S. Predictors of adherence to home rehabilitation exercises following anterior cruciate ligament reconstruction. Rehabil Psychol 2013;58:64-72..

35. BU Nwachukwu, Joshua Adjei, Ryan C. Rauck, Jorge Chahla, KR Okoroha, Verma NN, Allen AA, Riley J. Williams. How Much Do Psychological Factors Affect Lack of Return to Play After Anterior Cruciate Ligament Reconstruction? A Systematic Review Orthop J Sports Med. 2019 May 22;7(5).

\section{Tables}

TABLE 1. Demographic characteristics of groups 


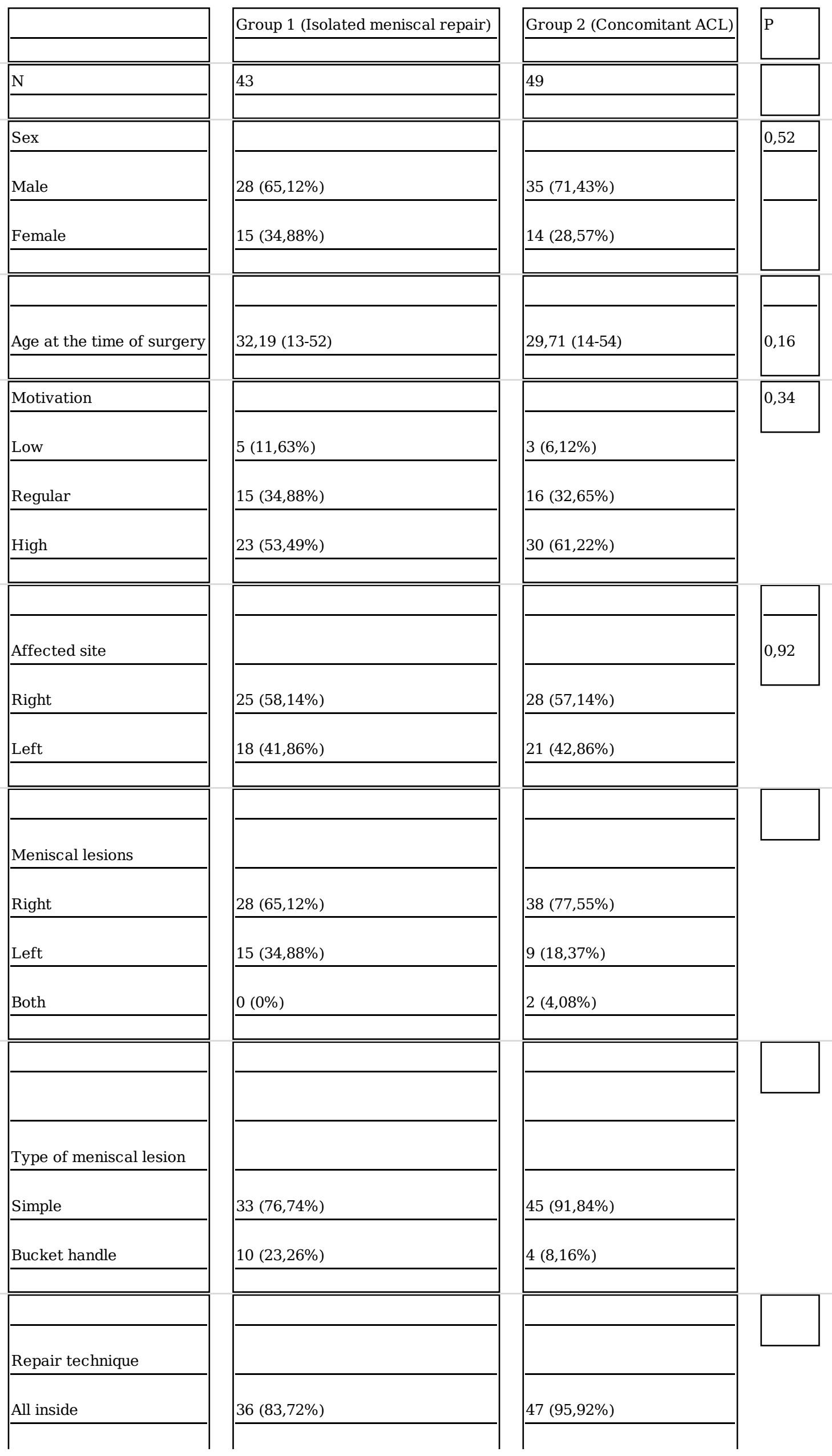

Page 18/24 


\begin{tabular}{|c|c|c|c|}
\hline Both & $7(16,28 \%)$ & $2(4,08 \%)$ & \\
\hline No. of sutures used & & & \\
\hline 1 & $9(20,93 \%)$ & $19(38,78 \%)$ & \\
\hline $2-3$ & $28(65,12 \%)$ & $24(48,98 \%)$ & \\
\hline 4 or more & $6(13,95 \%)$ & $6(12,24 \%)$ & \\
\hline Chondral lesions & $3(6,98 \%)$ & $1(2,04 \%)$ & 0,22 \\
\hline Reoperation & $7(16,28 \%)$ & $5(10,12 \%)$ & $<0,001$ \\
\hline Second surgery & & & \\
\hline Meniscectomy & $7(100 \%)$ & $4(80 \%)$ & \\
\hline Debridement & 0 & $1(20 \%)$ & \\
\hline
\end{tabular}

Table 2. COMPARATIVE MENISCAL FAILED SUTURE VS SUCCESFULL RECONSTRUCTION 


\begin{tabular}{|c|c|c|c|}
\hline & FAILED MENISCAL SUTURE & SUCCESFULL MENISCAL REPAIR & $\mathrm{P}$ \\
\hline sex & - & - & $\underline{0,19}$ \\
\hline MALE & $\underline{10}(83,33 \%)$ & $\underline{53}(\underline{66,25 \%})$ & \\
\hline FEMALE & $\underline{2}(\underline{16,67 \%})$ & $27(33,75 \%)$ & \\
\hline AGE & $\underline{29,92}$ & 31,01 & $\underline{0,67}$ \\
\hline MOTIVATION & - & - & $\underline{0,52}$ \\
\hline LOW & $3(\underline{25 \%})$. & $\underline{5}(\underline{6,25 \%})$ & \\
\hline REGULAR & $2(16,67 \%)$ & $29(36 ; 25 \%)$ & \\
\hline HIGH & 7 (58,33\%). & $\underline{46}(\underline{57,5 \%})$ & \\
\hline Knee side & - & - & $\underline{0,95}$ \\
\hline right & 7 (41,67\%). & $\underline{46}(\underline{57,5 \%})$ & \\
\hline left & $\underline{5(58,33 \%)}$ & $34(\underline{42,5 \%})$ & \\
\hline injured meniscus & - & - & $\underline{0,67}$ \\
\hline internal & $\underline{9}(\underline{75 \%})$. & $57(\underline{71,25 \%)}$. & \\
\hline external & $3(\underline{25 \%})$ & $21(26,25 \%)$ & \\
\hline both & & $2(2,5 \%)$. & \\
\hline type of meniscal lesion & - & - & $\underline{0,0062}$ \\
\hline simple & - & - & \\
\hline bucket handle & 7 (58,33\%). & 71 (88,75\%) & \\
\hline & 5 (41,67\%). & $\underline{9}(\underline{11,25 \%})$. & \\
\hline repair technique & - & - & - \\
\hline all inside & $\underline{9}(\underline{75 \%})$. & $74(\underline{92,5 \%})$ & $\underline{0,057}$ \\
\hline both & $\underline{3}(\underline{25 \%})$. & $\underline{6}(\underline{7,5 \%})$. & $\underline{0,29}$ \\
\hline no. sutures used & - & - & - \\
\hline & - & - & $\underline{0,68}$ \\
\hline 1 & $\underline{2}(\underline{16,67 \%})$. & $26(\underline{32,5 \%})$ & - \\
\hline $2-3$ & $\underline{8}(\underline{66,67 \%})$ & $\underline{44}$ (55\%) & \\
\hline 4 or $>$ & $2 \underline{2}(\underline{16,67 \%})$. & $10(\underline{12,5 \%})$. & \\
\hline chondral lesions & $1(8,33 \%)$ & $3(3,75 \%)$ & $\underline{0,49}$ \\
\hline LYSHOLM & 83,92 & 87,31 & $\underline{0,51}$ \\
\hline
\end{tabular}




\begin{tabular}{|c|c|}
\hline SF12-MCS & $\underline{55,33}$ \\
\hline SF12-PCS & $\underline{50,5}$ \\
\hline TEGNER & - \\
\hline PREOP & 5,33 \\
\hline POSTOP & $\underline{4,08}$ \\
\hline DURATION FROM INJURY TO SURGERY (MONTHS) & L \\
\hline & 10,92 \\
\hline
\end{tabular}

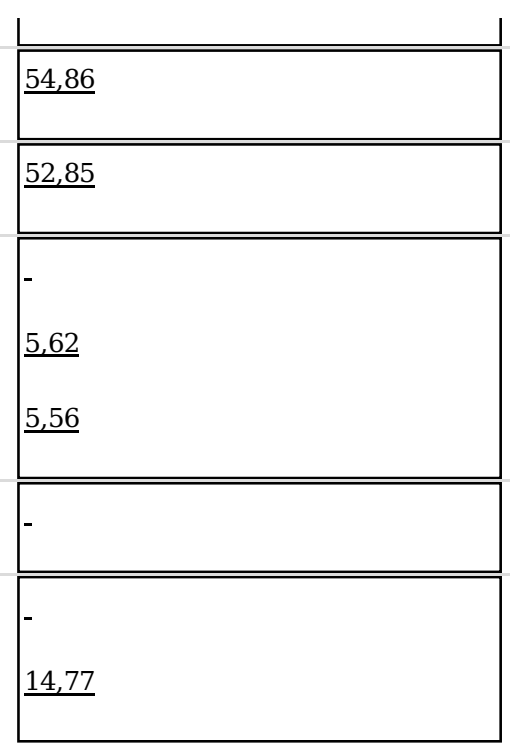

\begin{tabular}{|l|}
\hline$\underline{0,81}$ \\
\hline$\underline{0,4}$ \\
\hline- \\
\hline$\underline{0,48}$ \\
\hline$\underline{0,001}$ \\
\hline \hline- \\
\hline \hline- \\
\hline$\underline{0,12}$ \\
\hline
\end{tabular}

Table 3. COMPARATIVE PATIENT CHARACTERISTICS

Group 1 Isolated meniscal repair $\underline{\text { Group } 2 \text { Concomitant ACL }} \underline{P}$

$-$

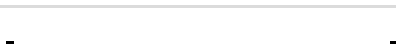

Duration from injury to surgery_(months).

$11,74+/-11,28$

$16,49+/-13,30$

0,06

Lysholm score

$89,35+/-10,66$

$84,7+/-16,10$

$89,35+/-10,66$

$84,7+/-16,10$

0,10

$-$

$\underline{\text { SF12-PCS }}$

$52,51+/-8,5$

$52,57+/-7,01$

0,97

$\underline{\text { SF12-MCS }}$

$55,83+/-8,09$

$54,12+/-7,77$

0,30

$-$

Tegner scale

$\underline{\text { Preoperative }}$

$5,11+/-1,37$

$6+/-0,98$

0,59

Postoperative

$5,46+/-1,44$

$5,31+/-1,43$ 


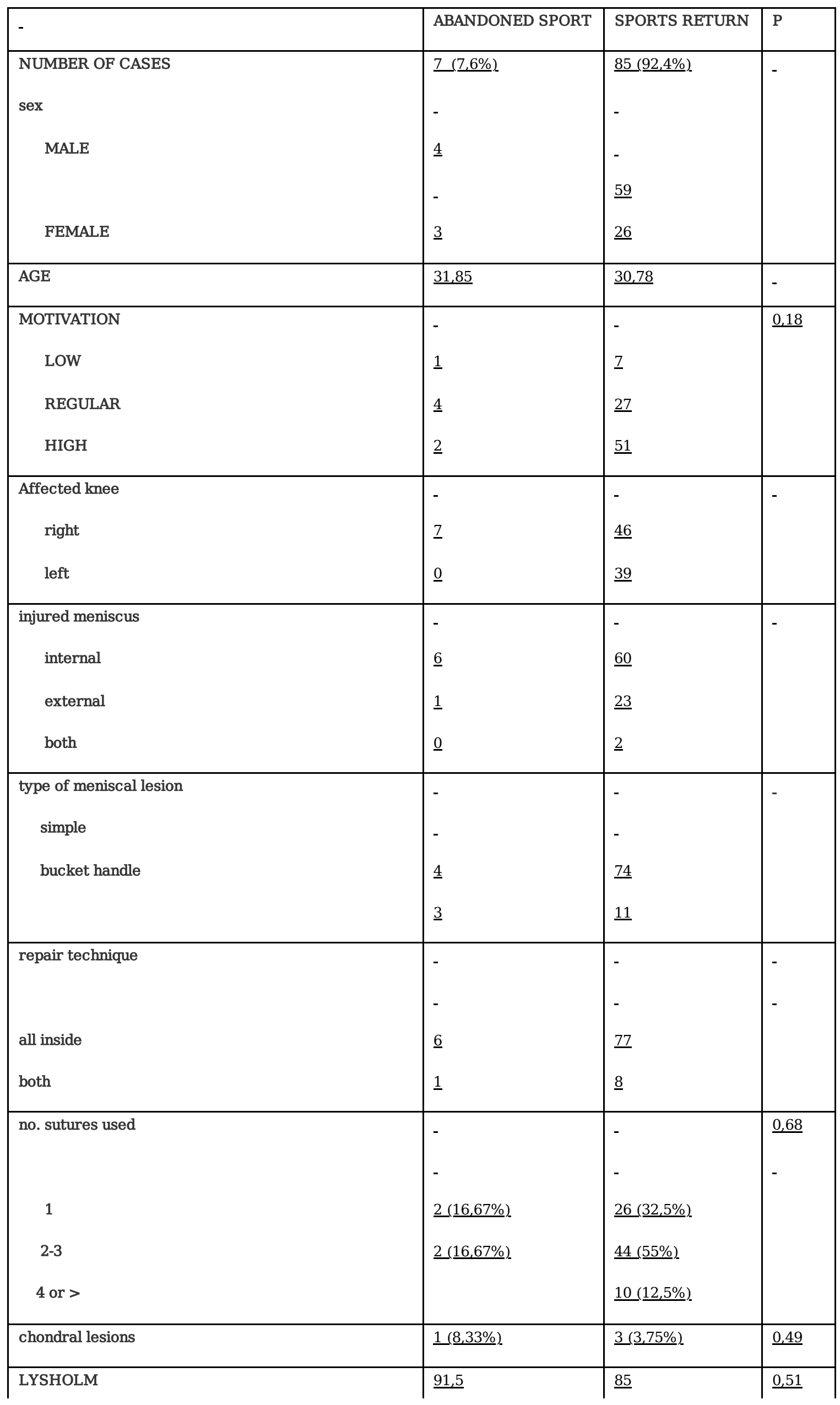

Page 23/24 


\begin{tabular}{|c|c|c|c|}
\hline SF12-MCS & $\underline{54,65}$ & $\underline{58,14}$ & $\leq 0,001$ \\
\hline SF12-PCS & $\underline{52,72}$ & $\underline{50,43}$ & $\leq 0,001$ \\
\hline TEGNER & _ & _ & _ \\
\hline PREOP & $\underline{5,33}$ & $\underline{5,62}$ & $\underline{0,48}$ \\
\hline \multirow[t]{2}{*}{ POSTOP } & $\underline{4,08}$ & $\underline{5,56}$ & $\underline{0,001}$ \\
\hline & - & - & - \\
\hline \multirow[t]{2}{*}{ DURATION FROM INJURY TO SURGERY (MONTHS) } & - & & - \\
\hline & $\underline{10,92}$ & $\underline{14,77}$ & $\underline{0,12}$ \\
\hline GROUP & - & - & - \\
\hline ACL+meniscal repair & $\underline{5}$ & $\underline{44}$ & $\underline{0,38}$ \\
\hline \multirow[t]{2}{*}{ Isolated meniscal repair } & - & - & \\
\hline & $\underline{7}$ & $\underline{36}$ & $\underline{0,38}$ \\
\hline
\end{tabular}

